\title{
Optica
}

\section{Haldane quantum Hall effect for light in a dynamically modulated array of resonators: supplementary material}

\author{
MOMCHIL MinKoV* AND Vincenzo SAVONA
}

Laboratory of Theoretical Physics of Nanosystems, Ecole Polytechnique Fédérale de Lausanne (EPFL), CH-1015 Lausanne, Switzerland *Corresponding author: momchil.minkov@epfl.ch

Published 17 February 2016

This document provides supplementary information to "Haldane Quantum Hall effect for light in a dynamically modulated array of resonators," http://dx.doi.org/10.1364/optica.3.000200. The Floquet theory used to obtain the quasi-energy bands of our time-periodic system is presented. Some further details on the numerical simulations are also given. (c) 2016 Optical Society of America

http://dx.doi.org/10.1364/optica.3.000200.s001

\section{Floquet theory}

We start from the most general, linear, time-independent Hamiltonian for photons on a lattice $(\hbar=1)$ :

$$
H_{S}=H_{0}+H_{J}=\sum_{i} \omega_{i} a_{i}^{\dagger} a_{i}-\sum_{i \neq j} J_{i j} a_{i}^{\dagger} a_{j}
$$

with $a_{i}^{\dagger}$, the photon creation operator, and no particular requirements for the couplings $J_{i j}$ (these will come later depending on the chosen lattice geometry). We add an on-site, time-dependent, periodic modulation of the resonant frequency with a positiondependent intensity and phase

$$
H_{t}=\sum_{i} A_{i} \cos \left(\Omega t+\phi_{i}\right) a_{i}^{\dagger} a_{i} .
$$

For times much larger than the period $T=2 \pi / \Omega$, it becomes meaningful to apply the Floquet theory of quasi-energies [1, 2]. In particular, the solutions to the time-dependent Schrödinger equation can be written as $\left|\psi_{n}(t)\right\rangle=\exp \left(-i \varepsilon_{n} t\right)\left|u_{n}(t)\right\rangle$, with $u_{n}(t)$ a $T$-periodic function which is a solution to

$$
\left[H_{S}+H_{t}-i \partial_{t}\right]\left|u_{n}(t)\right\rangle=\varepsilon_{n}\left|u_{n}(t)\right\rangle
$$

The spectrum $\varepsilon_{n}$ has a Brillouin-zone like structure with $\Omega$ the width of the first zone, i.e. for any solution $\left|u_{n}(t)\right\rangle$ of quasienergy $\varepsilon_{n}$, and any integer $m, \exp (i m \Omega t)\left|u_{n}(t)\right\rangle$ is also a solution, with eigenvalue $\varepsilon_{n}+m \Omega$. The states $\left|u_{n}(t)\right\rangle$ form a Hilbert space of $T$-periodic functions. The inner product in this space can be defined starting from the standard bra-ket inner product $\langle\bullet \mid \bullet\rangle$ for time-independent states, and reads

$$
\langle\langle\bullet \mid \bullet\rangle\rangle T \int_{0}^{T} \mathrm{~d} t\langle\bullet \mid \bullet\rangle,
$$

The states can be expanded on the Floquet basis given by

$$
\begin{aligned}
& \left|\left\{n_{i}\right\}, m\right\rangle=U_{t}(t)\left|\left\{n_{i}\right\}\right\rangle \exp (i m \Omega t) \\
= & \left|\left\{n_{i}\right\}\right\rangle \exp \left(-\frac{i}{\Omega} \sum_{i} A_{i} \sin \left(\Omega t+\phi_{i}\right) n_{i}+i m \Omega t\right),
\end{aligned}
$$

where $n_{i}$ denotes the occupation number of site $i$, and

$$
U_{t}(t)=\exp \left(-i \int_{t_{0}}^{t} H_{t}\left(t^{\prime}\right) \mathrm{d} t^{\prime}\right)
$$

is the time-evolution operator corresponding to the timedependent Hamiltonian $H_{t}$, and we assume an adiabatic switching of the modulation in order to disregard the phase offset due to the starting time $t_{0}$. Since the Hamiltonian is particlenumber-preserving, we need only consider the subspace of a single excitation in the system, $\sum_{i} n_{i}=1 \forall\left\{n_{i}\right\}$. Equation (S3) is then an eigenvalue problem with matrix elements in this basis given by

$$
\begin{gathered}
\left\langle\left\langle\left\{n_{j}^{\prime}\right\}, m^{\prime}\left|H_{S}+H_{t}-i \partial_{t}\right|\left\{n_{i}\right\}, m\right\rangle\right\rangle= \\
\delta_{m, m^{\prime}}\left[\left\langle\left\{n_{j}^{\prime}\right\}\left|H_{0}+m \Omega\right|\left\{n_{i}\right\}\right\rangle\right]+ \\
\int_{0}^{T} e^{i\left(m-m^{\prime}\right) \Omega t} \exp \left(\frac{i A_{j}}{\Omega} \sin \left(\Omega t+\phi_{j}\right)-\frac{i A_{i}}{\Omega} \sin \left(\Omega t+\phi_{i}\right)\right) \\
\times\left\langle\left\{n_{j}\right\}\left|H_{J}\right|\left\{n_{i}\right\}\right\rangle .
\end{gathered}
$$

These matrix elements are then equal to

$$
\left\langle\left\langle n_{i}^{\prime}, m^{\prime}\left|H_{S}+H_{t}-i \partial_{t}\right| n_{i}, m\right\rangle\right\rangle=\delta_{m, m^{\prime}}\left(m \Omega+\omega_{i}\right),
$$


when the particle stays on the same site, and to

$$
\begin{gathered}
\left\langle\left\langle n_{j}^{\prime}, m^{\prime}\left|H_{S}+H_{t}-i \partial_{t}\right| n_{i \neq j}, m\right\rangle=\int_{0}^{T} e^{i\left(m-m^{\prime}\right) \Omega t} J_{i j} \times\right. \\
\exp \left(\frac{i}{\Omega}\left(A_{j} \sin \left(\Omega t+\phi_{j}\right)-A_{i} \sin \left(\Omega t+\phi_{i}\right)\right)\right),
\end{gathered}
$$

when the particle hops from site $i$ to site $j$. In eqs. (S8) and (S9), we label by $n_{i}$ the only non-zero occupation number of $\left\{n_{i}\right\}$. We can further use the Jacobi-Anger expansion to simplify eq. (S9) to

$$
\begin{array}{r}
\left\langle\left\langle n_{j}^{\prime}, m^{\prime}\left|H_{S}+H_{t}-i \partial_{t}\right| n_{i \neq j}, m\right\rangle=\right. \\
\quad-\mathcal{J}_{m^{\prime}-m}\left(\rho_{i j}\right) e^{i\left(m^{\prime}-m\right) \phi_{i j}} J_{i j},
\end{array}
$$

with $\mathcal{J}_{n}(x)$ the $n$-th Bessel function of the first kind, and the definition

$$
\rho_{i j} e^{i \phi_{i j}}=\left(A_{j} e^{i \phi_{j}}-A_{i} e^{i \phi_{i}}\right) / \Omega \text {. }
$$

\section{Perturbation theory}

Some additional intuition can be found in writing the Floquet perturbation theory $[3,4]$ for the effective time-independent Hamiltonian $H_{\text {eff }}$ that describes the time evolution for timescales greater than $T$, in the sense that the time-evolution operator is

$$
\begin{aligned}
U(t+T, t) & =\exp \left(-i \int_{t}^{t+T}\left(H_{S}+H_{t}\right) \mathrm{d} t\right) \\
& =\exp \left(-i H_{\mathrm{eff}} T\right)
\end{aligned}
$$

For a Fourier-expanded time-periodic Hamiltonian,

$$
H(t)=\sum_{m} H_{m} e^{i m \Omega t},
$$

we can write a perturbation expansion for $H_{\text {eff }}$ in orders of $1 / \Omega$. Up to first order, this reads

$$
\begin{aligned}
H_{\mathrm{eff}} & =H_{0 \Omega}+H_{1 \Omega}+\mathcal{O}\left(\frac{1}{\Omega^{2}}\right) \\
& =H_{m=0}+\frac{1}{\Omega} \sum_{m=1}^{\infty} \frac{1}{m}\left[H_{m}, H_{-m}\right]+\mathcal{O}\left(\frac{1}{\Omega^{2}}\right) .
\end{aligned}
$$

In our dynamically-modulated lattice, after the unitary transformation

$$
\begin{gathered}
H^{\prime}=U_{t}^{\dagger}\left[H_{S}+H_{t}-i \partial_{t}\right] U_{t}=\sum_{i} \omega_{i} a_{i}^{\dagger} a_{i}- \\
\sum_{m} \sum_{i j} \mathcal{J}_{m}\left(\rho_{i j}\right) e^{i m\left(\Omega t+\phi_{i j}\right)} J_{i j} a_{i}^{\dagger} a_{j},
\end{gathered}
$$

with the definitions of $\mathcal{J}, \rho$, and $\phi$ as above, the Fourier components $H_{m}$ can be easily read out. The zero-th order of the perturbative expansion of eq. (S14) is

$$
\begin{aligned}
& H_{0 \Omega}=H_{0}+H_{J}^{\prime}=\sum_{i} \omega_{i} a_{i}^{\dagger} a_{i}-\sum_{i j} J_{i j}^{\prime} a_{i}^{\dagger} a_{j}, \\
& J_{i j}^{\prime}=J_{i j} \mathcal{J}_{0}\left(\rho_{i j}\right),
\end{aligned}
$$

i.e. similar to the starting $H_{S}$ of the static lattice, but with rescaled (but still real) couplings $J_{i j}^{\prime}$. The first-order term is

$$
\begin{aligned}
H_{1 \Omega}=\sum_{m=1}^{\infty} & \frac{(-1)^{m}}{\Omega m} \sum_{i j p q} \mathcal{J}_{m}\left(\rho_{i j}\right) \mathcal{J}_{m}\left(\rho_{p q}\right) J_{i j} J_{p q} e^{i m\left(\phi_{i j}-\phi_{p q}\right)} \\
& \times\left[a_{i}^{\dagger} a_{q} \delta_{j p}-a_{p}^{\dagger} a_{j} \delta_{i q}\right] \\
=\sum_{i j} 2 i \sum_{m=1}^{\infty} & \frac{(-1)^{m}}{\Omega m} \sum_{p} \mathcal{J}_{m}\left(\rho_{i p}\right) \mathcal{J}_{m}\left(\rho_{p j}\right) J_{i p} J_{p j} \\
& \times \sin \left(m\left(\phi_{i p}-\phi_{p j}\right)\right) a_{i}^{\dagger} a_{j}
\end{aligned}
$$

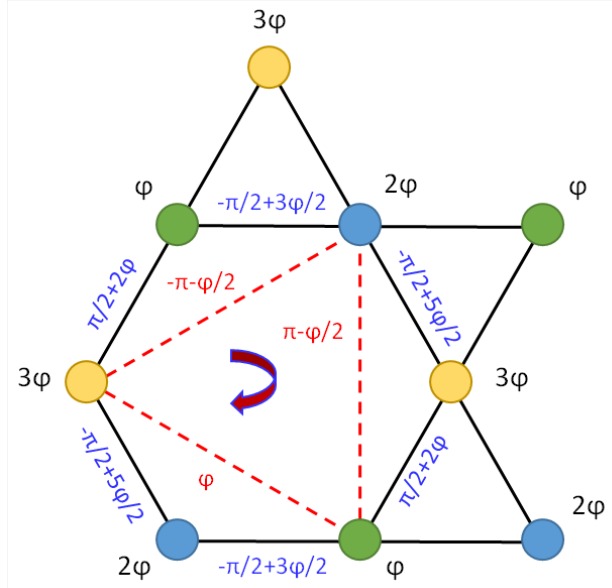

Fig. S1. Definition of various phases under a modulation of constant amplitude, and phases (marked in black): $\varphi$ on site A, $2 \varphi$ on site B, and $3 \varphi$ on site $C$ (see also to Fig. 2 in the main text). In blue, the phase $\phi_{i j}$ as defined in eq. (S11) is given for a clockwise hopping direction (indicated by the arrow in the center of the hexagon). With red, the phase $\phi_{i p}-\phi_{p j}$ entering eq. (S16) is given, for the same hopping direction.

The (purely imaginary) term after the first sum sign can obviously be interpreted as a new coupling amplitude, $J_{i j}^{\prime \prime}$, which is added to $J_{i j}^{\prime}$, thus introducing a complex phase. The obvious interpretation of these terms is hopping from one site to another through one intermediate site. In the same way, terms of higher order in $1 / \Omega$ represent hopping through an increasing number of intermediate sites.

\section{Kagomé lattice}

The Kagomé lattice (illustrated in Fig. S1 here and Fig. 2 of the main text) has three lattice sites in the unit cell, which we label A, B, and C. The values of the couplings of eq. (11) in the main text computed through (eq. (S16)) are

$$
\begin{array}{rl}
t_{A C, 0} & =\mathcal{J}_{0}\left(2 \rho_{\varphi}\right), \quad t_{A B, 0}=t_{B C, 0}=\mathcal{J}_{0}\left(\rho_{\varphi}\right) \\
t_{A B, 1} & =t_{A B, 1}^{\prime}= \\
2 & i \frac{J}{\Omega} \sum_{m} \frac{(-1)^{m}}{m} \mathcal{J}_{m}\left(\rho_{2 \varphi}\right) \mathcal{J}_{m}\left(\rho_{\varphi}\right) \sin (m(\varphi / 2-\pi)) \\
t_{A C, 1} & =t_{A C, 1}^{\prime}= \\
2 & i \frac{J}{\Omega} \sum_{m} \frac{(-1)^{m}}{m} \mathcal{J}_{m}\left(\rho_{\varphi}\right) \mathcal{J}_{m}\left(\rho_{\varphi}\right) \sin (m \varphi) \\
t_{B C, 1} & =t_{B C, 1}^{\prime}= \\
2 i & \frac{J}{\Omega} \sum_{m} \frac{(-1)^{m}}{m} \mathcal{J}_{m}\left(\rho_{2 \varphi}\right) \mathcal{J}_{m}\left(\rho_{\varphi}\right) \sin (m(\pi+\varphi / 2)),
\end{array}
$$

where $\rho_{\varphi}=2\left(A_{0} / \Omega\right)|\sin (\varphi / 2)|$ and $\rho_{2 \varphi}=2\left(A_{0} / \Omega\right)|\sin (\varphi)|$ are the amplitudes computed through eq. (S16) for a phase difference between sites $i$ and $j$ of $\varphi$ and $2 \varphi$, respectively. In Fig. $S 1$, we illustrate the computation of the phases that enter the sine functions of eq. (S17). Starting from the modulation phases (marked in black), one first computes the value of $\phi_{i j}$ (marked in blue) as defined in eq. (S11) for all first neighbors, and then the values of $\phi_{i p}-\phi_{p j}$ (marked in red) that enter eq. (S16).

Numerical simulations

We note that this perturbation theory discussion is only used for a better intuitive understanding of the effect, but the topo- 

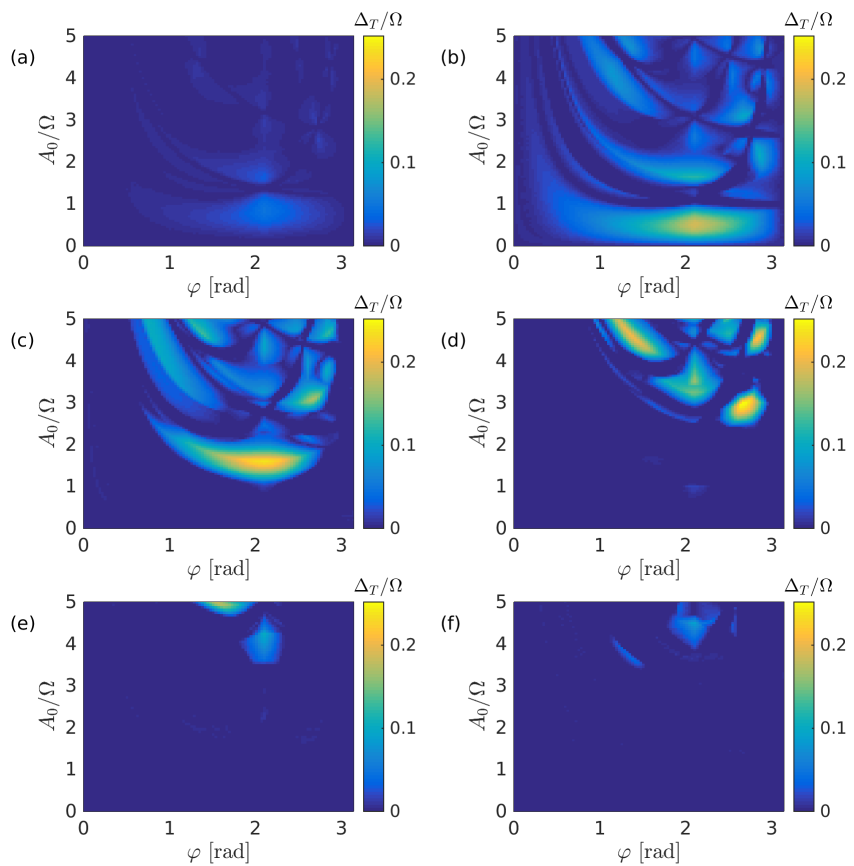

Fig. S2. The (largest) width of the opened band gap due to the dynamic modulation of frequency $\Omega$ vs. the amplitude $A_{0}$ and the phase angle $\varphi$ for the Kagomé lattice with first-neighbor coupling (a): $J=0.1 \Omega$; (b): $J=0.3 \Omega ;(\mathrm{c}): J=0.5 \Omega$; (d): $J=0.7 \Omega$; (e): $J=0.9 \Omega$; (f): $J=1.1 \Omega$. The colorbar scale is the same in all panels.

logically non-trivial bands are present even for values of $J$ that are comparable to $\Omega$. This is why in our work we mostly use the full diagonalization on the Floquet basis, i.e. we diagonalize the matrix of eq. (S7). Numerically, we truncate the orders of $m \Omega$ by picking an $m_{\max }$ value and taking orders up to that, i.e. $|m|,\left|m^{\prime}\right| \leq m_{\max }$. Convergence with respect to this parameter was always checked, and is reached very fast $\left(m_{\max } \approx 2\right)$ for low values of $J$ when the different orders of the Floquet bands are well separated in frequency space. As $J$ becomes comparable to $\Omega$, higher orders are needed, but convergence was always reached at $m_{\max }=10$, at most. The diagonalization of the effective static Hamiltonian (from perturbation theory) was only used for panels (b) of Fig. 2 and panels (a) and (c) of Fig. 3 of the main text. As is seen and can be expected, this matches the exact Floquet diagonalization very well for low $J / \Omega$, but becomes inadequate for higher values. Of course, that could in principle be fixed by higher orders in the perturbation expansion, but that would bring no further insight and is thus not really needed.

In Fig. S2 we show the width of the opened band gap $\Delta_{T}$ versus $A_{0}$ and $\varphi$, for $J$ from $0.1 \Omega$ to $1.1 \Omega$, computed by diagonalization on the Floquet basis. Panels (a) and (c) are shown in Fig. 3 of the main text (panels (b) and (d), respectively), but with a different scale of the colorbar. As $J$ increases, the amplitude $A_{0}$ needed to open a gap grows as well. This can at least qualitatively be understood through the following considerations. As $J$ becomes comparable to $\Omega$, Floquet bands of different orders $m$ start crossing, thus closing any potential band gaps. As $A_{0}$ increases, however, the re-scaled first-neighbor coupling $J_{i j}^{\prime}$ of eq. (S15) generally decreases, since $\rho_{i j}$ is proportional to $A_{0}$, and the Bessel function $\mathcal{J}_{0}(x)$ has its maximum value at $x=0$. Thus, the problem with mixing of bands of different orders can be avoided through a sufficiently large $A_{0}$.

\section{REFERENCES}

1. J. H. Shirley, "Solution of the schrödinger equation with a hamiltonian periodic in time," Phys. Rev. 138, B979-B987 (1965).

2. A. Eckardt, C. Weiss, and M. Holthaus, "Superfluid-insulator transition in a periodically driven optical lattice," Physical Review Letters 95, 1-4 (2005).

3. H. Sambe, "Steady states and quasienergies of a quantummechanical system in an oscillating field," Phys. Rev. A 7, 2203-2213 (1973).

4. G. Jotzu, M. Messer, R. Desbuquois, M. Lebrat, T. Uehlinger, D. Greif, and T. Esslinger, "Experimental realisation of the topological Haldane model," Nature 515, 237-240 (2014). 